Review

www.mdpi.com/journal/molecules

\title{
Chemical and Pharmacological Aspects of Capsaicin
}

\author{
Maria de Lourdes Reyes-Escogido *, Edith G. Gonzalez-Mondragon and \\ Erika Vazquez-Tzompantzi
}

Centro de Investigación y Asistencia en Tecnología y Diseño del Estado de Jalisco, A.C., Unidad Sureste, Calle 30 No. 151 por 7 y 7A, García Gineres, Mérida, 97070 Yucatán, Mexico

* Author to whom correspondence should be addressed; E-Mail: 1reyes@ciatej.net.mx; Tel.: +52-999-9202671; Fax: +52-999-9202671.

Received: 18 November 2010; in revised form: 9 January 2011 / Accepted: 25 January 2011 / Published: 28 January 2011

\begin{abstract}
Capsaicin is a unique alkaloid found primarily in the fruit of the Capsicum genus and is what provides its spicy flavor. Generally extracted directly from fruit, high demand has driven the use of established methods to increase production through extraction and characterization. Over time these methods have improved, usually be applying existing techniques in conjunction. An increasingly wide range of potential applications has increased interest in capsaicin. Especially compelling are the promising results of medical studies showing possible beneficial effects in many diseases. Capsaicin's pungency has limited its use in clinical trials to support its biological activity. Characterization and extraction/ synthesis of non-pungent analogues is in progress. A review is made of capsaicin research focusing mainly on its production, synthesis, characterization and pharmacology, including some of its main potential clinical uses in humans.
\end{abstract}

Keywords: capsaicin; capsinoids; synthesis; pharmacology

\section{Introduction}

Compounds known as capsaicinoids cause the spicy flavor (pungency) of chili pepper fruit. The primary capsaicinoid in chili pepper is capsaicin, followed by dihydrocapsaicin, nordihydrocapsaicin, homodihydrocapsaicin and homocapsaicin. Capsaicin and dihydrocapsaicin account for approximately $90 \%$ of capsaicinoids in chili pepper fruit, are the two most potent capsaicinoids and their molecules differ only in the saturation of the acyl group [1-3]. Capsaicin (trans-8-methyl- $N$-vanillyl-6nonenamide) is a crystalline, lipophilic, colorless and odorless alkaloid with the molecular formula 
$\mathrm{C}_{18} \mathrm{H}_{27} \mathrm{NO}_{3}$. Its molecular weight is $305.40 \mathrm{~g} / \mathrm{mol}$, and it is fat-, alcohol- and oil-soluble. First crystallized in 1876 by Tresh, who named it, capsaicin's molecular structure was resolved by Nelson and Dawson in 1919 [4]. Capsaicin displays cis/trans isomerism because the double bond prevents internal rotation. Capsaicin is always found as the trans isomer because in the cis form, the $-\mathrm{CH}\left(\mathrm{CH}_{3}\right)_{2}$ and the longer chain on the other side of the double bond will be close together, causing them to repel each other slightly; this steric hindrance does not exist in the trans isomer. This additional strain imposed causes the cis isomer to be a less stable arrangement than the trans isomer (Figure 1) [5].

Figure 1. Regions of the molecule of capsaicin. A (aromatic ring); B (amide bond); and C (hydrophobic side chain).

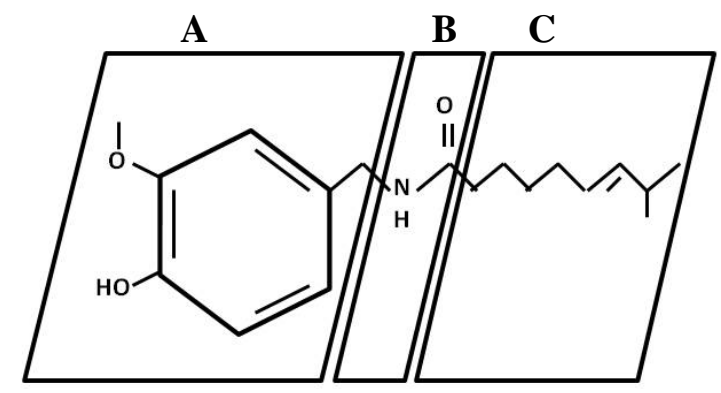

Structure-activity relationships (SAR) for capsaicin agonists (a substance that is capable of binding to a receptor and elicit a response in the cell), have previously been rationalized by dividing the capsaicin molecule into three regions: A (aromatic ring), B (amide bond) and C (hydrophobic side chain) (Figure 1). It is known that the substituents in the positions 3 and 4 of the A-ring are essential for potent agonist activity, and the phenolic 4-OH group in capsaicin analogues is of particular importance, H-bond donor/acceptor properties of the phenol group are key for agonist activity [6]. $\mathrm{C}$-Region SAR in capsaicin analogues have been discussed in detail in some reports. In summary, a hydrophobic group, e.g., an octyl chain or substituted benzyl or group, is required for high potency. Optimally, such aralkyl groups are substituted in the para position by small hydrophobic moieties. [2], by other way Barbero et al., [7] reported the importance of lateral chain lengths for the bioactivity of capsaicinoids, which was higher between 8 and 9 carbons atoms.

The more than twenty known capsaicinoids are all amides formed from condensation of vanillylamine and fatty acids of different chain lengths. The forms of different natural capsaicinoids depend on the number of lateral chain carbons (R) or the presence or absence of unsaturations (Figure 2). Capsaicinoids are synthesized naturally in the placenta of chili fruits from enzymatic condensation of vanillylamine and different-sized fatty acid chains which are elongated by a fatty acid synthase. This condensation is caused by the capsaicin synthase enzyme (CS) acting specifically on fatty acid chain length, which requires $\mathrm{Mg}^{2+}$, ATP and coenzyme A (CoA) and vanillylamine, the phenolic portion, formed from phenylalanine as a product in the phenylpropanoid pathway, while the fatty acid is formed from the amino acids valine or leucine. Structural differences among the capsaicinoids are defined by the nature of the lateral chain, which ranges from 9 to 11 carbons long with a variable number of double bonds located in different positions along the chain (Figure 2). 
Figure 2. Chemical structure of different capsaicinoids and their analogues. $\mathrm{R}$ Capsaicinoids and analogues.

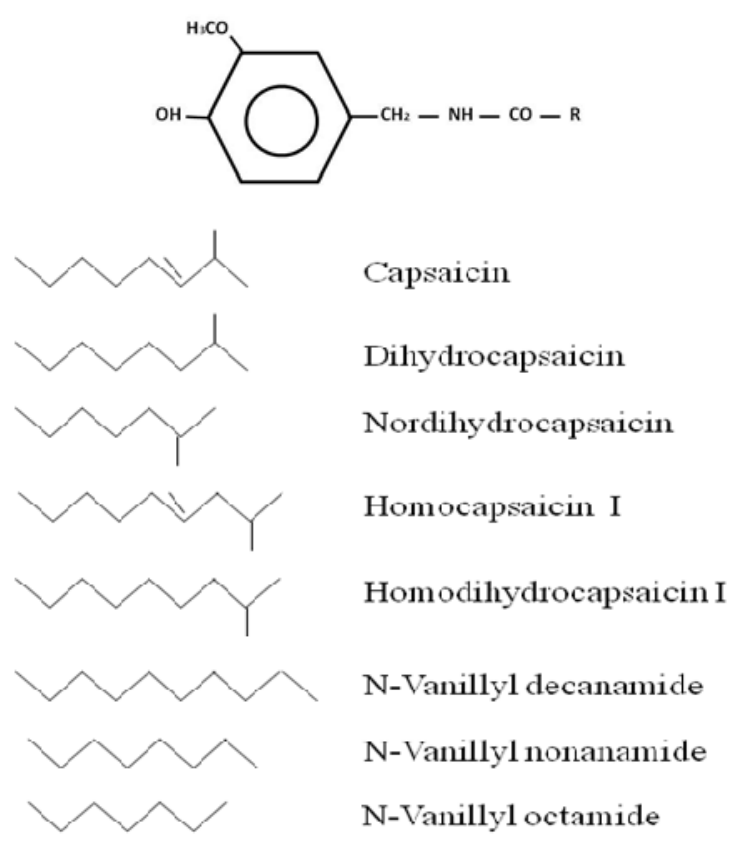

Capsaicinoids are important in the food and pharmaceutical industries. For this reason, a number of researchers are engaged in improving their production be it by manipulating chili plant cultivation conditions [8], chemical synthesis [9], enzymatic synthesis [10-11] or alternative methods such as cell or tissue culture [12]. To date, research has shown that capsaicinoids, and capsaicin in particular, have a wide variety of biological and physiological activities which provide them functions such as antioxidants [13], anticarcinogenics [14], promotion of energy metabolism and suppression of fat accumulation [15], and anti-inflammatories [16]. However, the potential applications of these molecules are limited by the irritation caused by their pungency; this has driven the search for and characterization of analogous molecules without inherent and undesirable effects [14,17-18].

\section{Capsaicin Production and Synthesis}

\subsection{Genetic Regulation of Capsaicin Synthesis}

Given the economic and agricultural importance of capsaicin, surprisingly little data exists on the genetics of this compound's biosynthesis pathway. It is known that chili genotypes exhibit wide variation in capsaicin accumulation in response to genetic and environmental factors [19]. The first genetic analysis of capsaicin accumulation was done by molecular mapping and revealed the presence of a Quantitative Trait Locus (QTL) called cap that may contribute to increasing pungency level [20]. Most of the isolated genes related to capsaicinoids are involved in their biosynthesis, and little is known about the location and action of specific genes controlling capsaicin accumulation. Transcript accumulation has been reported for the genes Pal, Ca4h and Comt, corresponding to the 
phenylpropanoid pathway [21], and the genes Kas, Acl and Fat, which code for the enzymes involved in fatty acid metabolism [22]. Spiciness is known to be inherited as a dominant trait and is associated with the Pun1 locus [23]. The product of Pun1 is AT3, corresponding to a putative acyltransferase, and specific to the placenta in pungent genotypes. Its highest expression coincides with capsaicinoid accumulation, meaning Pun1 exhibits a dominant behavior which determines pungent genotype occurrence [24]. Initially, it was thought that AT3 could correspond to CS. However, the csy1 gene was isolated and found to code for CS [25], and its sequence is different from that of AT3, indicating that Pun1 does not codify for CS. It was eventually established that the product of Pun1 is related to development of the vesicles where capsaicinoids accumulate and not to their production, although formation of these vesicles is vital for pungent phenotype occurrence [26].

\subsection{Capsaicin Biosynthesis in Plants}

Capsaicin biosynthesis in plants is defined by two pathways: phenylpropanoid, which determines phenolic structure; and fatty acid metabolism, which determines the molecule's fatty acids [27]. Capsaicin concentration increases gradually during fruit development reaching maximum levels at 40 to 50 days [28], after which it tends to degrade into secondary compounds due to peroxidase action [29]. Research aimed at increasing or improving pungent compound production has revealed that hydric stress increases capsaicinoid levels because water deficit affects the phenylpropanoid pathway [30]. Hydric stress also increases capsaicin levels by raising activity of the enzymes phenylalanine ammonia-lyase (PAL), cinnamic acid-4-hydroxylase $(\mathrm{C} 4 \mathrm{H})$ and $\mathrm{CS}$, all involved in capsaicin biosynthesis [8]. Administration of the capsaicin precursors 8-methyl-noneic acid and vanillylamine had demonstrated that 8-methylnoneic acid occurs at lower levels than vanillylamine and is therefore the limiting substrate for capsaicin synthesis [31]. These results suggest the possibility of controlling capsaicin synthesis in the plant by manipulating substrate concentrations and water availability, which would be a cost-effective, viable alternative for increasing capsaicin production.

\subsection{Chemical and Enzymatic Synthesis}

Interest in capsaicinoids has increased in response to their multiple properties, mainly those with potential medical applications [32]. However, clinical application of capsaicin is limited because of it pungent properties. In response, greater research effort has been applied to synthesizing natural capsaicinoids as well as synthetics with properties analogous to natural types [17].

Enzyme-catalyzed synthesis has advantages over chemical synthesis in that it can be done using nontoxic reagents and substrate specificity [33]. Kobata and colleagues did a number of studies involving synthesis of capsaicin analogues using amidation reactions catalyzed with different lipases [34]. In these trials, vanillylamine condensed with fatty acid derivatives, used as a substrate in the oleose phase, resulted in 40-59\% capsaicin yields and synthesis of various analogues at $2-44 \%$ yields and with from 4-18 carbons [35]. This same research group used natural oils as substrates in different reaction media (e.g. n-hexane) to synthesized non-pungent analogues [34].

Castillo and colleagues synthesized non-pungent analogues using different acyl chain lengths and chemical substitutes in the aromatic ring. They obtained two pungent analogues and other very low pungency analogues with potential uses (Figure 3) [10-11]. 
Figure 3. Synthesis of capsaicin analogues using different amines and donors as substrates.

\begin{tabular}{|c|c|c|c|c|c|}
\hline Compound & $\mathrm{X}_{1}$ & $\mathbf{X}_{2}$ & Compound & & $\mathbf{R}$ \\
\hline 1 & $\mathrm{MeO}-$ & $\mathrm{HO}-$ & A & - & $\mathrm{C}_{3} \mathrm{H}_{7}$ \\
\hline 2 & $\mathrm{H}-$ & $\mathrm{H}-$ & B & - & $\mathrm{C}_{5} \mathrm{H}_{11}$ \\
\hline \multirow[t]{6}{*}{3} & $\mathrm{MeO}-$ & $\mathrm{H}-$ & $\mathrm{C}$ & - & $\mathrm{C}_{7} \mathrm{H}_{15}$ \\
\hline & & & D & - & $\mathrm{C}_{9} \mathrm{H}_{19}$ \\
\hline & & & $\mathrm{E}$ & - & $\mathrm{C}_{11} \mathrm{H}_{23}$ \\
\hline & & & $\mathrm{F}$ & - & $\mathrm{C}_{13} \mathrm{H}_{27}$ \\
\hline & & & G & - & $\mathrm{C}_{15} \mathrm{H}_{31}$ \\
\hline & & & $\mathrm{H}$ & & $\begin{array}{l}\mathrm{C}\left(\mathrm{CH}_{2}\right)_{7} \mathrm{CH}- \\
\mathrm{CH}\left(\mathrm{CH}_{2}\right)_{7} \mathrm{CH}_{3}\end{array}$ \\
\hline
\end{tabular}

Capsaicin and its analogs are produced industrially using chlorinated fatty acids and amines at temperatures between 140 and $170{ }^{\circ} \mathrm{C}$ under moderate pressure [36]. A number of studies have been done using chemical synthesis of capsaicinoids. Choi and Yoon [37] used bioisosterism to synthesized 1-hydroxy-2-pyridone, a capsaicin analogue with similar biological effects. A study using chemoselective esterification of phenolic acids with alcohols for vanillyl nonanoate synthesis involved reactions between vanillyl alcohol and nonanoic acid using tetrahydrofurane as reaction medium and equimolar amounts of diisopropyl azodicarboxylate and triphenyl phosphine. Run at room temperature for $24 \mathrm{~h}$, the reaction produced a 67\% vanillyl nonanoate yield [38]. In another study, cerium chlorate (III) was used as a reaction catalyst in selective esterification of phenolic alcohols, resulting in a $70 \%$ vanillyl nonanoate yield [39]. Research has also been done on combined chemical-enzymatic synthesis of the non-pungent analogue dihydronorcapsaicin $\beta$-D-glucopyranoside [40]. The limited success of chemical synthesis of capsaicin is due to the toxicity of the required reagents, a disadvantage which makes enzymatic synthesis an appealing alternative to traditional chemical synthesis.

Capsinoids other analogues of capsaicin were recently identified from the non pungent type of red pepper CH-19 sweet, being the principal capsinoids the capsiate, dihydrocapsiate and nordihydrocapsiate [41]. Capsinoids has a remarkable structural resemblance to capsaicinoids except for their center linkage: an amide moiety in capsaicinoid and an ester moiety in capsinoid (Figure 4). Studies have shown that bio-potency of capsinoids is similar to capsaicin without the pungency or sensory irritation. Although the interest of some groups has been focused in these molecules [17,42], until now their mechanisms of action are poorly understood. These compounds are found naturally in peppers, but only in a few varieties. To obtain a sufficient volume of these capsaicinoids they will need to be synthesized, however the presence of the terminal methyl group and double bond complicates their synthesis and increases the cost [7]. 
Figure 4. Structures of capsaicin and capsiate. Capsiate has an ester bond, whereas capsaicin has an amide bond at the equivalent position.

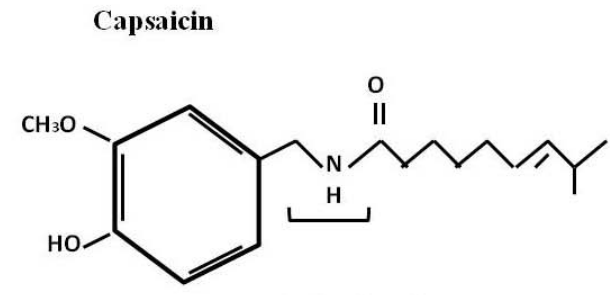

Amine bond
Capsiate

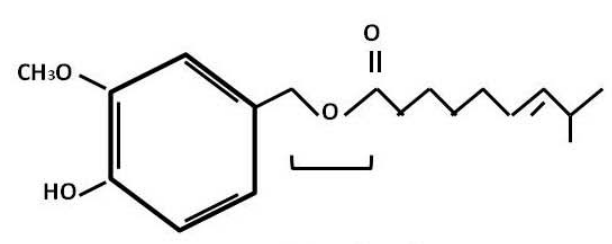

Ester bond

\subsection{In Vitro Synthesis}

A promising alternative for synthesis of capsaicin and its analogues is in vitro synthesis. Capsaicinoid production via cell or tissue culture can be augmented be addition of biosynthetic pathway precursors and intermediaries as phenylalanine, ferulic acid and vanillylamine showing encouraging results [43]. Cell suspensions were employed to study the influence of phenylalanine and other phenylpropanoids, in this study the addition of $100 \mu \mathrm{M}$ of either phenylalanine, cinnamic acid or caffeic acids did not cause significant increment in capsaicinoids content during the growth cycle. But the addition of $100 \mu \mathrm{M}$ of vanillin, vanillylamine, $p$-coumaric acid and ferulic acid did increase 10, 7.5, 5.2 and 2.5 fold higher the capsaicin production as compared to control, respectively [44]. Other compounds such as coumaric acid and elicitors such as phycocyanin, synapinic acid, salicylic acid and curdlan result in different capsaicin production levels [45]. Accumulation of capsaicinoids and the intermediary vanillylamine have also been reported with use of the inducers salicylic acid and methyl jasmonate in cell suspension cultures [46]. Likewise, administration of the supplements L-ascorbic acid and D-limonene in the culture medium produced an approximately three-fold increase in capsaicin production [47]. A 45\% increase in capsaicin production has been reported using callus cultures resistant to $p$-fluorophenylalanine [48]. Addition of the polyamine putrescine to suspension cell cultures has been shown to increase capsaicin production and activity of capsaicin synthase, the capsaicin biosynthesis terminal enzyme [49].

In vitro capsaicin production has been more efficient in immobilized cell cultures than in cell suspension cultures because the former use precursors for capsaicin synthesis whereas the latter use them for primary metabolism. This was demonstrated in a comparative study of both culture types using radioactively-marked phenylalanine and cinnamic acid to monitor their incorporation into capsaicin and/or its precursors. Incorporation of both compounds was higher in the immobilized cell cultures than in the suspension cultures because in the suspension cultures the phenylalanine and cinnamic acid were incorporated into protein metabolism and the cell wall, resulting in lower capsaicin production [50]. The same phenomenon has been reported for placental tissue cultures, with higher capsaicin production in immobilized versus suspension cultures; this is to be expected to some degree since the placenta contains the biochemical machinery needed for capsaicin synthesis [51]. In other study using cultures derived from placenta of Capsicum annuum L. which were tried with 100\% nitrogen stress increased capsaicin level by 9.8 fold of that in control after 15 days of subculturing. Addition of $4 \mathrm{mM}$ tyrosine to $100 \%$ phosphorus and $100 \%$ potassium stress led to 8.4 fold and 7.5 fold 
higher capsaicin production as compared with the control, respectively. They used various precursors too; $4 \mathrm{mM}$ coumaric acid, $5 \mathrm{mM}$ ferulic acid, and $4 \mathrm{mM}$ vanillin which showed 6.3, 4.7 and 2.4 fold increases, respectively, being coumaric acid the most effective. Finally, they used the elicitors phycocyanin $(0.25 \%)$, sinapic acid $(0.05 \mathrm{mM})$, salicylic acid $(2 \mathrm{mM})$ and curdlan $(0.0625 \%)$, being the sinapic acid the most effective which increased capsaicin up to 8.9 fold in the cell suspension cultures. Although salicylic acid and curdlan had not effect, curdlan in combination with tyrosine, increased the accumulation of capsaicin 8.7 fold, and had an effect in the phenylalanine ammonialyase activity [45].

Putative capsaicin precursors and analogues isolated from Capsicum placenta and cell suspension cultures transform products into capsaicin glycoside and other identified compounds. It is also possible that peroxidases from Capsicum fruit and cell suspension cultures produce capsaicinoids. In vitro culture is clearly a promising alternative for capsaicin production since it allows for control of culture conditions and is scalable.

\section{Capsaicin Characterization}

Growing interest in capsaicin has led to its characterization with methods such as spectrophotometry UV-VIS and chromatography. These have been modified over time to develop more sensitive, faster capsaicin characterization techniques.

Thin-layer chromatography has been used to detect capsaicin on ground pepper [52], and multi-band thin-layer chromatography has been used to evaluate the $\mathrm{R}_{\mathrm{f}}$ of capsaicin in different adsorbents and the same mobile phase under identical conditions (i.e. time, temperature, solvent vapor equilibrium, etc.) [53]. High-performance liquid chromatography (HPLC) has been widely used for characterization of capsaicin and it analogues [54-59]. Using different detectors, such as fluorescence, HPLC has been used to study capsaicinoids [60]. For example, this method has been applied to detect capsaicin, zucapsaicin and civamide (a capsaicin cis-isomer) within a 1 to $100 \mathrm{ng} / \mathrm{mL}$ concentration interval [61], and to characterize six capsaicin analogues up to a $3 \mathrm{ng}$ concentration in animal tissues and blood [62]. This method was also used to quantify air-borne capsaicin and dihydrocapsaicin at a pickling and pepper manufacturing plant. Capsaicin concentration was 0.015 and dihydrocapsaicin concentration was $0.02 \mu \mathrm{g}$ per sample; the capsaicin:dihydrocapsaicin ratio remained within a $0.3: 1$ to 0.5:1 range, not coinciding with the higher capsaicin content in Capsicum [63]. Storage stability in capsaicin solutions has been monitored using HPLC during a one year period, in a concentration range of 0.5 to $128 \mu \mathrm{M}$ and four temperature/light combinations: $4{ }^{\circ} \mathrm{C}$ in darkness; room temperature in darkness; room temperature in light; and $-20{ }^{\circ} \mathrm{C}$ in darkness. Under the tested conditions, the $4 \mu \mathrm{M}$ treatment exhibited optimum performance, remaining stable for one year when stored at $4{ }^{\circ} \mathrm{C}$ in darkness [64]. In another study, HPLC with a diode array detector allowed quantification of capsaicin at levels below 100 Scoville units (the Scoville unit indicates the amount of capsaicin present) when mixed with nordihydrocapsaicin and dihydrocapsaicin, and at levels below 1000 Scoville units when mixed with spices such as cumin and pepper [65]. Reverse-phase HPLC was applied to identify and quantify four lipidic hydroperoxide isomers produced by oxidation of linoleic acid to evaluate capsaicin's antioxidant activity [66].

Technological advances in the use of HPLC for organic compound analysis have been applied to capsaicin characterization, specifically liquid chromatography-mass spectrometry (LC-MS) [67-69] 
and liquid chromatography-tandem mass spectrometry (LC-MS-MS) [70]. The latter method was used to analyze capsaicinoid content in commercial pepper sprays with a standard quantification curve within a 10 to $1,000 \mathrm{ng} / \mathrm{mL}$ concentration interval. Capsaicin, dihydrocapsaicin and nonivamide were identified in the sprays within a 0.7 to $40.5 \mathrm{mg} / \mathrm{mL}$ interval [71]. In other applications, LC-MS-MS has been used to quantify capsaicin, dihydrocapsaicin and nonivamide in the blood and tissues of rats exposed to inhalations of these three compounds with resulting capsaicin concentrations of 1.0 to $90.4 \mathrm{ng} / \mathrm{mL}$ in blood, 5.0 to $167 \mathrm{pg} / \mathrm{mg}$ in lungs, and 2.0 to $3.4 \mathrm{pg} / \mathrm{mg}$ in liver [72]. Simultaneous quantification of capsaicin and dihydrocapsaicin in extracts from different Capsicum genotypes has been accomplished using liquid chromatography-electrospray/time-of-flight mass spectrometry with detection limits of 6 ng for capsaicin and $1.2 \mathrm{ng}$ for dihydrocapsaicin [73-74]. Liquid chromatography quadrupole ion trap mass spectrometry (LC-ESI/MS/MS) has been successfully applied to quantify capsaicin's in vitro half-life (2.3 to $4.1 \mathrm{~min}$ ) [75], as well as blood capsaicin content in rats subcutaneously administered capsaicin and dihydrocapsaicin [76].

In a same way gas chromatography (GC) has been used in the study of capsaicin [77]. In a comparison of capsaicin detection using GC and HPLC, the detection limit was $1.0 \mu \mathrm{g} / \mathrm{g}$ with a linear capsaicin calibration curve of 1 to $250 \mu \mathrm{g} / \mathrm{mL}$ with $\mathrm{GC}$ and 0.5 to $50 \mu \mathrm{g} / \mathrm{mL}$ with HPLC [78]. The two methods have also been used in tandem (GCL) for determining the pungency in raw spices, extracts, food and pharmaceutical products. The pungency data obtained by both methods, the sensory test and CGL, ranged from 50,000 to $2,000,000$ Scoville units with a correlation coefficient of 0.95 by a period of one year [79-80].

Solid phase microextraction-gas chromatography-mass spectrometry is another method used to study capsaicinoids [81]. In an analysis of eleven pepper varieties and four pepper-based sauces, use of this method did not require derivatization, which is required with GC, and it effectively identified and quantified capsaicin $(55-25,459 \mu \mathrm{g} / \mathrm{g})$ and dihydrocapsaicin $(93-1,130 \mu \mathrm{g} / \mathrm{g})$ in the peppers $(4.3-717.3 \mu \mathrm{g} / \mathrm{g})$ and sauces $(1.0-134.8 \mu \mathrm{g} / \mathrm{g})$ [82].

A modification of capillary chromatography that has been applied to capsaicin and dihydrocapsaicin in different varieties of Capsicum frutescens, is the micellar electrokinetic capillary chromatography. In this method, samples are separated by differential partition between micelles (pseudo stationary stage) and the mobile phase (aqueous buffer at $\mathrm{pH}$ 9). Both capsaicin and dihydrocapsaicin were detected within $11 \mathrm{~min}$ with an excellent resolution [83].

Capsaicin's hydrophobic nature suggests that it may influence membrane structure, which was studied using the dipalmitoylphosphatidylcholine and dielaidoylphosphatidylethanolamine membrane models and the differential scanning calorimetry, fluorescent probe spectroscopy and ${ }^{31} \mathrm{P}$-nuclear magnetic resonance techniques. The results were used to propose the possible implications of capsaicin's effects in biological membranes [84].

Proton nuclear magnetic resonance $\left({ }^{1} \mathrm{H}-\mathrm{NMR}\right)$ and carbon nuclear magnetic resonance $\left({ }^{13} \mathrm{C}-\mathrm{NMR}\right)$ were used in a comparative analysis of capsaicinoids purity and structure [85]. ${ }^{1} \mathrm{H}-\mathrm{NMR}$ has also been used in quantitative analysis of capsaicin in Capsicum frutescens $L$. without previous derivatization [86]. ${ }^{13} \mathrm{C}$-NMR, ${ }^{1} \mathrm{H}$-NMR and $\alpha$ - and $\beta$-glucosydase hydrolysis were used to identify the structure of two capsaicin glucosides: capsaicin- $\beta$-D-glucopyranoside and dihydrocapsaicin- $\beta$-D-glucopyranoside. In this study, the glucosides were not detected in non-pungent $C$. annum crops and a positive correlation was observed between capsaicin and dihydrocapsaicin levels and their glucosides [87]. 
The UV-VIS spectrophotometric method is one of the most inexpensive and accessible for capsaicin quantification; indeed, most laboratories have a UV-VIS spectrophotometer. However, analysis is restricted to capsaicin solutions with microgram-level concentrations [88].

Kachoosangi et al. [89] discuss capsaicin detection methods other than chromatography and spectroscopy with detection ranges from 0.5 to $35 \mu \mathrm{M}$, while capsaicin's molecular and electronic structure can be analyzed using the density functional theory [90].

\section{Capsaicin Pharmacology}

\subsection{Pharmacokinetics}

Capsaicin is known to be effectively absorbed topically from the skin. In a study of 12 subjects topically administered a $3 \%$ capsaicin solution in three different vehicles $(70 \%$ isopropyl alcohol; mineral oil; and propylene glycol in $20 \%$ alcohol), capsaicin was shown to be rapidly absorbed and to quickly reach maximum concentration when capsaicin is applied topically. The half-life of capsaicin was approximately $24 \mathrm{~h}$ [91].

In an extensive study of capsaicin distribution in tissues, elimination and other active principles in animals with oral administration, nearly $94 \%$ of orally administered capsaicin was absorbed and maximum concentration in the blood was reached $1 \mathrm{~h}$ after administration. In addition, a maximum distribution of $24.4 \%$ of administered capsaicin in blood, liver, kidney and intestine was seen in $1 \mathrm{~h}$ and then diminished notably until being undetected after 4 days [92].

Capsaicin metabolism is apparently similar in human, rat and dog microsome. Three major metabolites have been identified for capsaicin: 16-hydroxycapsaicin, 17-hydroxycapsaicin and 16,17dihydrocapsaicin [93]. In vitro studies in human skin, capsaicin biotransformation was found to be slow and most capsaicin remained unchanged while a small fraction was metabolized to vanillylamine and vanillyl acid. This suggests that cytochrome $\mathrm{P}_{450}$ enzymes participate minimally in capsaicin transformation in skin in comparison to their role in hepatic metabolism. Capsaicin is mainly eliminated by the kidneys with a small untransformed proportion excreted in the feces and urine [92,94].

\subsection{Mechanism of Action}

From the past decade the mechanism of action of capsaicin has been extensively studied. Capsaicin can bond to transient receptor potential vanilloid 1 (TRPV1), previously known as the vanilloid receptor, which is mainly expressed in the sensory neurons [95]. First identified and cloned in rats, TRPV1 contains 838 amino acids and has a molecular weight of $95 \mathrm{kDa}$ in both humans and rats, consisting of six transmembrane domains with a short pore-forming region between the fifth and sixth transmembrane domains [96]. This receptor is a non-selective, ligand-operated cationic channel located primarily in the small fibers of nociceptive neurons. TRPV1 is also broadly distributed in tissues of the brain, bladder, kidneys, intestines, keratinocytes of epidermis, glial cells, liver, and polymorphonuclear granulocytes, mast cells, and macrophages [95,97]. It couples with a non-specific cation channel permeable to sodium and calcium ions, and is located in the plasma membrane and the endoplasmic reticulum where regulates intracellular calcium levels [98-99]. This channel can be regulated and activated by endogenous substance as endovanilloids and diverse exogenous stimuli 
including chemical agonists as capsaicin, olvanil and resiniferatoxin, ligands highly lipophilic that share structural similarity to several endogenous fatty acids identified as TRPV1 agonists [100]. Someone compounds are being used as antagonists for TRPV1, including capsazepine, iodoresiniferatoxin, ruthenium red, A-425619, SB-366791, AMG9810, and SB-705498 [101]. The TRPV1 contains a heat-sensitive subunit responsible for the burning sensation caused by capsaicin. Bonding of capsaicin to TRPV1 increases intracellular calcium, triggering release of neuropeptides such as substance $\mathrm{P}$ and the calcium gene-related peptide (CGRP). Contact between capsaicin and sensory neurons produces pain, inflammation and a localized heat sensation. When applied locally to skin, it promotes an analgesic response due to desensitizing of the sensory neurons caused by substance $\mathrm{P}$ depletion [102]. This mechanism has served as a base for studies of the structure-activity relationship which are aimed at development of new synthetic ligands for the TRPV1. Capsaicin's effects in the nervous system are not exclusively analgesic. A number of studies include results showing that it participates in release of somatostatine, CGRP and endotheline [103-107].

\subsection{Clinical Applications in Humans}

Capsaicin has been attributed pharmacological effects since ancient times, but not until the past twenty years has extensive research been done to determine specific applications, including in the gastrointestinal tract, for weight-loss and as an analgesic.

Research into the possible protective role of capsaicin in the gastrointestinal tract have produced controversial results and clearly indicates the need for further clinical trials to better define effective dosages. At low concentrations $(0.13-160 \mu \mathrm{M})$ in rats, capsaicin is reported to protect the gastric mucous against ulceration by ethanol [108], whereas when administered at high concentrations ( 1 or $2 \mathrm{mg} / \mathrm{mL}$ ) in rat stomachs it is reported to worsen damage to the gastric mucous caused by ethanol or aspirin [109]. In a study involving 84 healthy human subjects, capsaicin was found to have protective properties against ethanol- and indomethacine-associated gastropathy, with a dosedependent decrease in base gastric acid output ( $\mathrm{ED}_{50}$ for $400 \mu \mathrm{g}$ capsaicin) and increased gastric emptying [110]. A possible explanation is that capsaicin mediates the antiulcerous effect by vasodilation and increasing gastric mucous blood flow (GMBF) and this is mediated by nitric oxide and CGRP release by these TRPV1 bearing cells [110].

Nearly 20 years ago, another major pharmacological effect of capsaicin was identified when it was found that it reduced adipose tissue in rodents by enhancing energy and lipid metabolism, possibly by increasing catecholamine secretion from the adrenal medulla in response to activation of the sympathetic nervous system [111-112]. These findings have helped to support further research into capsaicin's possible therapeutic effects and mechanisms in obesity treatment.

As mentioned above, capsaicin has been included in topical treatments aimed at relief of different neuropathic pain conditions, although it can produce skin irritation. Capsaicin and its analogues have been used in topical creams and patches to treat chronic pain syndromes such as post-herpetic neuralgia, musculoskeletal pain, diabetic neuropathy, osteoarthritis and rheumatoid arthritis [113-114]. It has also been applied to treat pain from rashes, psoriasis, mastectomy, and bladder disorders. Adverse effects (burning, stinging and erythema) are normally limited to the application site, although respiratory irritations from cream inhalation and occasional systemic effects have been reported [115]. 
Its actual efficacy in pain relief is still somewhat in doubt due to the small number of participants and diverse definitions of pain used in studies. However, it is apparently a good alternative for pain relief in patients who do not respond to other therapies, be it when administered alone or together with other treatments [116].

\section{Conclusions}

Advances in analytical techniques are been used in the study capsaicin to increase the knowledge about its molecular characterization and structural-activity relationship. Low production yield has led to development of new synthesis strategies, including improving its biosynthesis in the plant by manipulating growth conditions or adding supplements. Other options are chemical and enzymatic synthesis or in vitro cell culture, all of which are promising for production of capsaicin and its analogues because they allow control of reactions to generate molecules with desired characteristics. Capsaicin is an important molecule in areas such as medicine but its pungency limits use in clinical trials. Non-pungent analog molecules have been developed in response. This is a promising molecule with many possible clinical applications but the paucity of clinical trials prevents its widespread pharmacological use.

\section{Acknowledgements}

This research was supported by grant No. 86988 from Fondo Sectorial de Investigación en Salud y Seguridad Social.

\section{References}

1. Bernal, M.A.; Calderon, A.A.; Pedreno, M.A.; Muñoz, R.; Ros Barceló, A.; Merino de Caceres, F. Capsaicin oxidation by peroxidase from Capsicum annuum (variety Annuum) fruits. J. Agric. Food Chem. 1993, 41, 1041-1044.

2. Walpole, C.S.; Bevan, S.; Bloomfield, G.; Breckenridge, R.; James, I.F.; Ritchie, T.; Szallasi, A.; Winter, J.; Wrigglesworth, R. Similarities and differences in the structure-activity relationships of capsaicin and resiniferatoxin analogues. J. Med. Chem. 1996, 39, 2939-2952.

3. Kobata, K.; Kawamura, M.; Toyoshima, M.; Tamura, Y.; Ogawa, S.; Watanabe, T. Lipasecatalyzed synthesis of capsaicin analogs by amidation of vanillylamine with fatty acid derivatives. Biotechnol. Lett. 1998, 20, 451-454.

4. Nelson, E.K.; Dawson, L.E. The constitution of capsaicin, the pungent principle of Capsicum. III. J. Am. Chem. Soc. 1923, 45, 2179-2181.

5. http://www.3dchem.com/molecules.asp?ID=105, accessed on 28 January 2011.

6. Katritzky, A.R.; Xu, Y.J.; Vakulenko, A.V.; Wilcox, A.L.; Bley, K.R. Model compounds of caged capsaicin: design, synthesis, and photoreactivity. J. Org. Chem. 2003, 68, 9100-9104.

7. Barbero, G.F.; Molinillo, J.M.G.; Varela, R.M.; Palma, M.; Macias, F.A.; Barroso, C.G. Application of Hansch's model to capsaicinoids and capsinoids: a study using the quantitative structure-activity relationship. A novel method for the synthesis of capsinoids. J. Agric. Food Chem. 2010, 58, 3342-3349. 
8. Sung, Y.; Chang,Y.Y.; Ting, N.L. Capsaicin biosynthesis in water-stressed hot pepper fruits. Bot. Bull. Acad. Sin. 2005, 46, 35-42.

9. Kaga, H.; Miura, M.; Orito, K. A facile procedure for synthesis of capsaicin. J. Org.Chem. 1989, 54, 3477-3478.

10. Castillo, E.; Lopez-Gonzalez, I.; De Regil-Hernandez, R.; Reyes-Duarte, D.; Sánchez-Herrera, D.; López-Munguía, A.; Darszon, A. Enzymatic synthesis of capsaicin analogs and their effect on the T-type $\mathrm{Ca}^{2+}$ channels. Biochem. Biophys. Res. Comm. 2007, 356, 424-430.

11. Castillo, E.; Torres-Gavilán, A.; Severiano, P.; Arturo, N.; López-Munguía, A. Lipase-catalyzed synthesis of pungent capsaicin analogues. Food Chem. 2007, 100, 1202-1208.

12. Ochoa-Alejo, N.; Ramírez-Malagón, R. In vitro chili pepper biotechnology. In Vitro Cell. Dev. Biol. Plant. 2001, 37, 701-729.

13. Materska, M.; Perucka, I. Antioxidant activity of the main phenolic compounds isolated from hot pepper fruit (Capsicum annuum L.). J. Agric. Food Chem. 2005, 53, 1750-1756.

14. Macho, A.; Lucena, C.; Sancho, R.; Daddario, N.; Minassi, A.; Munoz, E.; Appendino, G. Nonpungent capsaicinoids from sweet pepper synthesis and evaluation of the chemopreventive and anticancer potential. Eur. J. Nutr. 2003, 42, 2-9.

15. Ohnuki, K.; Niwa, S.; Maeda, S.; Inoue, N.; Yazawa, S.; Fushiki, T. CH-19 sweet, a non-pungent cultivar of red pepper, increased body temperature and oxygen consumption in humans. Biosci. Biotechnol. Biochem. 2001, 65, 2033-2036.

16. Sancho, R.; Lucena, C.; Macho, A.; Calzado, M.A.; Blanco-Molina, M.; Minassi, A.; Appendino, G.; Muñoz, E. Immunosuppressive activity of capsaicinoids: capsiate derived from sweet peppers inhibits NF- $\mathrm{kB}$ activation and is a potent antiinflammatory compound in vivo. Eur. J. Immunol. 2002, 32, 1753-1763.

17. González Molinillo, J.M.; Macias Domínguez, F.A.; Varela Montoya, R.M.; Palma Lovillo, M.; García Barroso, C.; Fernández Barbero, G. Method for the chemical synthesis of capsinoids. US Patent 2010/0256413 A1.

18. Lida, T.; Moriyama, T.; Kobata, K.; Morita, A.; Murayama, N.; Hashizume, S.; Fushiki, T.; Yazawa, S.; Watanabe, T.; Tominaga, M. TRPV1 activation and induction of nociceptive response by a non-pungent capsaicin-like compound, capsiate. Neuropharmacology 2003, 44, 958-967.

19. Harvell, K.P.; Bosland, P.W. The environment produces a significant effect on pungency of chiles. Hort. Sci. 1997, 32, 1292.

20. Blum, E.; Mazourek, M.; O'Connell, M.; Curry, J.; Thorup, T.; Liu, K.; Jahn, M.; Paran, I. Molecular mapping of capsaicinoid biosynthesis genes and quantitative trait loci analysis for capsaicinoid content in Capsicum. Theor. Appl. Genet. 2003, 108, 79-86.

21. Curry, J.; Aluru, M.; Mendoza, M.; Nevarez, J.; Melendrez, M.; O'Connell, M.A. Transcripts for possible capsaicinoid biosynthetic genes are differentially accumulated in pungent and nonpungent Capsicum spp. Plant Sci. 1999, 148, 47-57.

22. Aluru, M.R.; Mazourek, M.; Landry L.G.; Curry, J.; Jahn, M.; O'Conell, M.A. Differential expression of fatty acid synthase genes, Acl, Fat and Kas, in Capsicum fruit. J. Exp. Bot. 2003, 54, 1655-1664. 
23. Andrews, J. Peppers: The Domesticated Capsicums; University of Texas Press: Austin, TX, USA, 1995; p. 274.

24. Stewart, C.; Kang, B.C.; Liu, K.; Mazourek, M.; Moore, S.L.; Yoo, E.Y.; Kim, B.D.; Paran, I.; Jahn, M.M. The pun1 gene for pungency in pepper encodes a putative acyltransferase. Plant J. 2005, 42, 675-688.

25. Prasad, B.C.; Kumar, V.; Gururaj, H.B.; Parimalan, R.; Giridhar, P.; Ravishankar, G.A. Characterization of capsaicin synthase and identification of its gene (csy1) for pungency factor capsaicin in pepper (Capsicum sp.). Proc. Natl. Acad. Sci. USA 2006, 103, 13315-13320.

26. Stewart, C.; Mazourek, M.; Stellari, G.M.; O'Connell, M.; Jahn, M. Genetic control of pungency in C. chinense via the Pun1 locus. J. Exp. Bot. 2007, 58, 979-991.

27. Ochoa-Alejo, N.; Gomez-Peralta, J.E. Activity of enzymes involved in capsaicin biosynthesis in callus tissue and fruits of chili pepper (Capsicum annuum L.) J. Plant Physiol. 1993, 141, 147-152.

28. Contreras-Padilla, M.; Yahia, E.M. Changes in capsaicinoids during development, maturation, and senescence of chile peppers and relation with peroxidase activity. J. Agric. Food Chem. 1998, 46, 2075-2079.

29. Bernal, M.A.; Ros Barceló, A. 5,5'- dicapsaicin, 4'-O-5-dicapsaicin ether, and dehydrogenation polymers with high molecular weights are the main products of the oxidation of capsaicin by peroxidase from hot pepper. J. Agric. Food Chem. 1996, 43, 352-355.

30. Estrada, B.; Pomar; F.; Díaz, J.; Merino, F.; Bernal, M.A. Pungency levels in fruits of the padron pepper with different water supply. Hort. Sci. 1999, 81, 385-396.

31. Prasad, N.C.; Gururaj, H.B.; Kumar, V.; Giridhar, P.; Parimalan, R.; Sharma, A.; Ravishankar, G.A. Influence of 8-methyl-nonenoic acid on capsaicin biosynthesis in in-vivo and in-vitro cell cultures of Capsicum spp. J. Agric. Food Chem. 2006, 54, 1854-1859.

32. Burch, R.M.; Carter, R.B.; Lazar, J. Injectable capsaicin. Patent US 2005/0019436 A1, 2005.

33. Iwai, K.;Watanabe, T.; Tamura, Y.; Ogawa, S. Method of producing capsaicin analogues. US Patent 6,022,718.

34. Kobata, K.; Kobayashi, M.; Tamura, Y.; Miyoshi, S.; Ogawa, S.; Watanabe, T. Lipase-catalyzed synthesis of capsaicin analogs by transacylation of capsaicin with natural oils or fatty acid derivatives in n-hexane. Biotechnol. Lett. 1999, 21, 547-550.

35. Kobata, K.; Toyoshima, M.; Kawamura, M.; Watanabe, T. Lipase-catalyzed synthesis of capsaicin analogs using natural oils as an acyl donor. Biotechnol. Lett. 1998, 20, 781-783.

36. Kaga, H.; Goto, K.; Takahashi, T.; Hino, M.; Tokuhashi, T.; Orito, K. A general and stereoselective synthesis of the capsaicinoids via the orthoester Claisen rearrangement. Tetrahedron 1996, 52, 8451-8470.

37. Choi, H.Y.; Yoon, S.H. Bioisoster of capsaicin: Synthesis of 1-hydroxy-2-pyridone analogue. Bull. Kor. Chem. Soc. 1999, 20, 857-859.

38. Appendino, G.; Minassi, A.; Morello, A.S.; De Petrocellis, L.; Di Marzo, V. N-acylvanillamides: Development of an expeditious synthesis and discovery of new acyl templates for powerful activation of the vanilloid receptor. J. Med. Chem. 2002, 45, 3739-3745.

39. Torregiani, E.; Seu, G.; Minassi, A.; Appendino, G. Cerium (III) chloride promoted chemoselective esterification of phenolic alcohols. Tetrahedron Lett. 2005, 46, 2193-2196. 
40. Sultana, I.; Shimamoto, M.; Obata, R.; Nishiyama, S.; Sugai, T. An expeditious chemo-enzymatic synthesis of dihydronorcapsaicin $\beta$-D-glucopyranoside. Sci. Technol. Adv. Mater. 2006, 7, 197-201.

41. Sutoh, K.; Kobata, K.; Yazawa, S.; Watanabe, T. Capsinoid is biosynthesized from phenylalanine and valine in a non-pungent pepper, Capsicum annuum L. cv. CH-19 sweet. Biosci. Biotechnol. Biochem. 2006, 70, 1513-1516.

42. Amino, Y.; Kurosawa, W.; Nakano, T.; Hirasawa, K. Production method of capsinoid by dehydratation condensation, stabilizing method of capsinoid, and capsinoid composition. US Patent 7700331.

43. Johnson, T.S.; Ravishankar, G.A.; Venkataraman, L.V. Biotransformation of ferulic acid and vanillylamine to capsaicin and vanillin in immobilized cell cultures of Capsicum frutescens. Plant Cell. Tiss. Organ. Cult. 1996, 44, 117-121.

44. Nuñez-Palenius, H.; Ochoa-Alejo, N. Effect of phenylalanine and phenylpropanoids on the accumulation of capsaicinoids and lignin in cell cultures of chili pepper (Capsicum annuum L.). In Vitro Cell. Dev. Biol. Plant. 2005, 41, 801-805.

45. Pandhair, V.; Gosal, S.S. Capsaicin production in cell suspension cultures derived from placenta of Capsicum annuum L. fruit. Indian J. Agric. Biochem. 2009, 22, 78-82.

46. Gutiérrez-Carbajal, M.; Monforte-González, M.; Miranda-Ham, M.; Godoy-Hernández, G.; Vázquez-Flota, F. Induction of capsaicinoid synthesis in Capsicum chinense cell cultures by salicylic acid or methyl jasmonate. Biol. Plant. 2010, 54, 430-434.

47. Veeresham C.; Kokate, C.K.; Apte, S.S.; Venkateshwarly, V. Effect of precursors on capsaicin Capsicum annuum. Plant Tiss. Cult. 1993, 3, 67-70.

48. Johnson, T.; Sarada, R.; Ravishankart, G. Capsaicin formation in p-fluorophenylalanine resistant and normal cell cultures of Capsicum frutescens and activity of phenylalanine ammonia lyase. J. Biosci. 1998, 23, 209-212.

49. Sudha, G.; Ravishankar, G.A. Putrescine facilitated enhancement of capsaicin production in cell suspension cultures of Capsicum frutescens. J. Plant Physiol. 2003, 160, 339-346.

50. Lindsey, K. Incorporation of $\left[{ }^{14} \mathrm{C}\right]$ phenylalanine and $\left[{ }^{14} \mathrm{C}\right]$ cinnamic acid into capsaicin in cultured cells of Capsicum frutescens. Phytochemistry 1986, 25, 2793-2801.

51. Johnson, T.S.; Ravishankar, G.A.; Venkataraman, L.V. In vitro capsaicin production by immobilized cells and placental tissues of Capsicum annuum L. grown in liquid medium. Plant Sci. 1990, 70, 223-229.

52. Spanyar, P.; Blazovich, M. A thin-layer chromatographic method for the determination of capsaicin in ground paprika. Analyst 1969, 94, 1084-1089.

53. Pankar, D.S.; Magar, N.G. New method for the determination of capsaicin by using multi-band thin-layer chromatography. J. Chromatogr. 1977, 144, 149-152.

54. Sato, K.; Sasaki, S.S.; Goda, Y.; Yamada, T.; Nunomura, O.; Ishikawa, K.; Maitani, T. Direct connection of supercritical fluid extraction and supercritical fluid chromatography as a rapid quantitative method for capsaicinoids in placentas of Capsicum. J. Agric. Food Chem. 1999, 47, 4665-4668.

55. Choi, S.H.; Suh, B.S.; Kozukue, E.; Kozukue, N.; Levin, C.E.; Friedman, M. Analysis of the contents of pungent compounds in fresh korean red peppers and in pepper-containing foods. J. Agric. Food Chem. 2006, 54, 9024-9031. 
56. Barbero, G.F.; Palma, M.; Barroso, C.G. Pressurized liquid extraction of capsaicinoids from peppers. J. Agric. Food Chem. 2006, 54, 3231-3236.

57. Barbero, G.F.; Palma, M.; Barroso, C.G. Determination of capsaicinoids in peppers by microwave-assisted extraction-high-performance liquid chromatography with fluorescence detection. Anal. Chim. Acta. 2006, 578, 227-233.

58. Barbero, G.F.; Liazid, A.; Palma, M.; Barroso, C.G. Ultrasound-assisted extraction of capsaicinoids from peppers. Talanta 2008, 75, 1332-1337.

59. Thapa, B.; Skalko-Basnet, N.; Takano, A.; Masuda, K.; Basnet, P. High-performance liquid chromatography analysis of capsaicin content in 16 Capsicum fruits from Nepal. J. Med. Food 2009, 12, 908-913.

60. Mueller-Seitz, E.; Hiepler, C.; Petz, M. Chili pepper fruits: content and pattern of capsaicinoids in single fruits of different ages. J. Agric. Food Chem. 2008, 56, 12114-12121.

61. Lu, J.; Cwik, M. Determination of capsaicin and zucapsaicin in human serum by highperformance liquid chromatography with fluorescence detection. J. Chromatogr. B Biomed. Sci. Appl. 1997, 701, 135-139.

62. Saria, A.; Lembeck, F.; Skofitsch, G. Determination of capsaicin in tissues and separation of capsaicin analogues by high-performance liquid chromatography. J. Chromatogr. 1981, 208, 41-46.

63. Tucker, S.P. Determination of capsaicin and dihydrocapsaicin in air in a pickle and pepper processing plant. AIHAJ 2001, 62, 45-48.

64. Kopec, S.E.; DeBellis, R.J.; Irwin, R.S. Chemical analysis of freshly prepared and stored capsaicin solutions: implications for tussigenic challenges. Pulm. Pharmacol. Ther. 2002, 15, 529-534.

65. Weaver, K.M.; Awde, D.B. Rapid high-performance liquid chromatographic method for the determination of very low capsaicin levels. J. Chromatogr. 1986, 367, 438-442.

66. Henderson, D.E.; Slickman, A.M.; Henderson, S.K. Quantitative HPLC determination of the antioxidant activity of capsaicin on the formation of lipid hydroperoxides of linoleic acid: a comparative study against BHT and melatonin. J. Agric. Food Chem. 1999, 47, 2563-2570.

67. Kozukue, N.; Han, J.S.; Kozukue, E.; Lee, S.J.; Kim, J.A.; Lee, K.R.; Levin, C.E.; Friedman, M. Analysis of eight capsaicinoids in peppers and pepper-containing foods by high-performance liquid chromatography and liquid chromatography-mass spectrometry. J. Agric. Food Chem. 2005, 53, 9172-9181.

68. Friedman, M.; Levin, C.E.; Lee, S.U.; Lee, J.S.; Ohnisi-Kameyama, M.; Kozukue, N. Analysis by HPLC and LC/MS of pungent piperamides in commercial black, white, green, and red whole and ground peppercorns. J. Agric. Food Chem. 2008, 56, 3028-3036.

69. Kobata, K.; Saito, K.; Tate, H.; Nashimoto, A.; Okuda, H.; Takemura, I.; Miyakawa, K.; Takahashi, M.; Iwai, K.; Watanabe, T. Long-chain $N$-vanillyl-acylamides from Capsicum oleoresin. J. Agric. Food Chem. 2010, 58, 3627-3631.

70. Srinivas, N.R. LC/MS/MS analysis of capsaicin using multiple transition pairs-some view points to ponder. Biomed. Chromatogr. 2009, 23, 1129-1130.

71. Reilly, C.A.; Crouch, D.J.; Yost, G.S.; Fatah, A.A. Determination of capsaicin, dihydrocapsaicin, and nonivamide in self-defense weapons by liquid chromatography-mass spectrometry and liquid chromatography-tandem mass spectrometry. J. Chromatogr. A 2001, 912, 259-267. 
72. Reilly, C.A.; Crouch, D.J.; Yost, G.S.; Fatah, A.A. Determination of capsaicin, nonivamide, and dihydrocapsaicin in blood and tissue by liquid chromatography-tandem mass spectrometry. J. Anal. Toxicol. 2002, 26, 313-319.

73. Garcés-Claver, A.; Arnedo-Andrés, M.S.; Abadía, J.; Gil-Ortega, R.; Alvarez-Fernandez, A. Determination of capsaicin and dihydrocapsaicin in Capsicum fruits by liquid chromatographyelectrospray/time-of-flight mass spectrometry. J. Agric. Food Chem. 2006, 54, 9303-9311.

74. Garcés-Claver, A.; Gil-Ortega, R.; Alvarez-Fernandez, A.; Arnedo-Andrés, M.S. Inheritance of capsaicin and dihydrocapsaicin, determined by HPLC-ESI/MS, in an intraspecific cross of Capsicum annuum L. J. Agric. Food Chem. 2007, 55, 6951-6957.

75. Beaudry, F.; Vachon, P. Quantitative determination of capsaicin, a transient receptor potential channel vanilloid 1 agonist, by liquid chromatography quadrupole ion trap mass spectrometry: evaluation of in vitro metabolic stability. Biomed. Chromatogr. 2009, 23, 204-211.

76. Zhang, Q.; Hu, J.; Sheng, L.; Li, Y. Simultaneous quantification of capsaicin and dihydrocapsaicin in rat plasma using HPLC coupled with tandem mass spectrometry. J. Chromatogr. B Analyt. Technol. Biomed. Life Sci. 2010, 878, 2292-2297.

77. Muller-Stock, F.A.; Joshi, R.K.; Buchi, J. Study of the components of capsaicin. Quantitative gas chromatographic determination of individual homologs and analogs of capsaicin in mixtures from a natural source and of vanillyl pelargonic amide as adulteration. J. Chromatogr. 1971, 63, 281-287.

78. Ha, J.; Han, K.J.; Kim, K.J.; Jeong, S.W. Gas chromatographic analysis of capsaicin in Gochujang. J. Assoc. Anal. Chem. Int. 2008, 91, 387-391.

79. Todd Jr, P.H.J.; Bensinger, G.; Biftu, T. Determination of pungency due to Capsicum by gasliquid chromatography. J. Food Sci. 1977, 42, 660-665.

80. DiCecco, J.J. Gas-liquid chromatographic determination of capsaicin. J. Assoc. Anal. Chem. 1976, 59, 1-4.

81. Thiele, R.; Mueller-Seitz, E.; Petz, M. Chili pepper fruits: presumed precursors of fatty acids characteristic for capsaicinoids. J. Agric. Food Chem. 2008, 56, 4219-4224.

82. Peña-Alvarez, A.; Ramirez-Maya, E.; Alvarado-Suarez, L.A. Analysis of capsaicin and dihydrocapsaicin in peppers and pepper sauces by solid phase microextraction-gas chromatography-mass spectrometry. J. Chromatogr. A 2009, 1216, 2843-2847.

83. Laskaridou-Monnerville, A. Determination of capsaicin and dihydrocapsaicin by micellar electrokinetic capillary chromatography and its application to various species of Capsicum, Solanaceae. J. Chromatogr. A 1999, 838, 293-302.

84. Aranda, F.J.; Villalain, J.; Gomez-Fernandez, J.C. Capsaicin affects the structure and phase organization of phospholipid membranes. Biochim. Biophys. Acta 1995, 1234, 225-234.

85. Peng, A.; Ye, H.; Li, X.; Chen, L. Preparative separation of capsaicin and dihydrocapsaicin from Capsicum frutescens by high-speed counter-current chromatography. J. Sep. Sci. 2009, 32, 2967-2973.

86. Nazari, F.; Ebrahimi, S.N.; Talebi, M.; Rassouli, A.; Bijanzadeh, H.R. Multivariate optimisation of microwave-assisted extraction of capsaicin from Capsicum frutescens L. and quantitative analysis by ${ }^{1} \mathrm{H}-\mathrm{NMR}$. Phytochem. Anal. 2007, 18, 333-340. 
87. Higashiguchi, F.; Nakamura, H.; Hayashi, H.; Kometani, T. Purification and structure determination of glucosides of capsaicin and dihydrocapsaicin from various Capsicum fruits. J. Agric. Food Chem. 2006, 54, 5948-5953.

88. Davis, C.B.; Markey, C.E.; Busch, M.A.; Busch, K.W. Determination of capsaicinoids in habanero peppers by chemometric analysis of UV spectral data. J. Agric. Food Chem. 2007, 55, 5925-5933.

89. Kachoosangi, R.T.; Wildgoose, G.G.; Compton, R.G. Carbon nanotube-based electrochemical sensors for quantifying the 'heat' of chilli peppers: the adsorptive stripping voltammetric determination of capsaicin. Analyst 2008, 133, 888-895.

90. Alberti, A.; Galasso, V.; Kovac, B.; Modelli, A.; Pichierri, F. Probing the molecular and electronic structure of capsaicin: a spectroscopic and quantum mechanical study. J. Phys. Chem. A 2008, 112, 5700-5711.

91. Pershing, L.K.; Reilly, C.A.; Corlett, J.L.; Crouch, D.J. Effects of vehicle on the uptake and elimination kinetics of capsaicinoids in human skin in vivo. Toxicol. Appl. Pharmacol. 2004, 200, 73-81.

92. Suresh, D.; Srinivasan, K. Tissue distribution and elimination of capsaicin, piperine and curcumin following oral intake in rats. Indian J. Med. Res. 2010, 131, 682-691.

93. Chanda, S.; Bashir, M.; Babbar, S.; Koganti, A.; Bley, K. In vitro hepatic and skin metabolism of capsaicin. Drug Metab. Dispos. 2008, 36, 670-675.

94. Kawada, T.; Iwai, K. In vivo and in vitro metabolism of dihydrocapsaicin, a pungent principle of hot pepper, in rats. Agric. Biol. Chem. 1985, 49, 441-448.

95. Cortright, D.N.; Szallasi, A. Biochemical pharmacology of the vanilloid receptor TRPV1. Eur. J. Biochem. 2004, 271, 1814-1819.

96. Caterina, M.J.; Schumacher, M.A.; Tominaga, M.; Rosen, T.A.; Levine, J.D.; Julius, D. The capsaicin receptor: a heat-activated ion channel in the pain pathway. Nature 1997, 389, 816-824.

97. Tominaga, M.; Tominaga, T. Structure and function of TRPV1. Pflugers Arch. 2005, 451, 143-150.

98. Liu, M.; Liu, M.C.; Magoulas, C.; Priestley, J.V.; Willmott, N.J. Versatile regulation of cytosolic $\mathrm{Ca}^{2+}$ by vanilloid receptor I in rat dorsal root ganglion neurons. J. Biol. Chem. 2003, 278, 5462-5472.

99. Kárai, L.J.; Russell, J.T.; Iadarola, M.J.; Oláh, Z. Vanilloid receptor 1 regulates multiple calcium compartments and contributes to $\mathrm{Ca}^{2+}$-induced $\mathrm{Ca}^{2+}$ release in sensory neurons. J. Biol. Chem. 2004, 279, 16377-16387.

100. Morita, A.; Iwasaki, Y.; Kobata, K.; Lida, T.; Higashi, T.; Oda, K.; Susuki, A.; Narukawa, M.; Sasakuma, S.; Yokogoshi, H.; Yazawa, S.; Tominaga, M.; Watanabe, T. Lipophilicity of capsaicinoids and capsinoids influences the multiple activation process of rat TRPV1. Life Sci. 2006, 79, 2303-2310.

101.Pingle, S.C.; Matta, J.A.; Ahern, G.P. Capsaicin receptor: TRPV1 a promiscuous TRP channel. Handb. Exp. Pharmacol. 2007, 179, 155-171.

102. Bevan, S.; Szolcsanyi, J. Sensory neuron-specific actions of capsaicin: mechanisms and applications. Trends Pharmacol. Sci. 1990, 11, 330-333.

103. Saria, A.; Lundberg, J.M.; Hua, X.; Lembeck, F. Capsaicin-induced substance P release and sensory control of vascular permeability in the guinea-pig ureter. Neurosci. Lett. 1983, 41, 167-172. 
104. Jhamandas, K.; Yaksh, T.L.; Harty, G.; Szolcsanyi, J.; Go, V.L. Action of intrathecal capsaicin and its structural analogues on the content and release of spinal substance P: selectivity of action and relationship to analgesia. Brain Res. 1984, 306, 215-225.

105. Purkiss, J.; Welch, M.; Doward, S.; Foster, K. Capsaicin-stimulated release of substance P from cultured dorsal root ganglion neurons: involvement of two distinct mechanisms. Biochem. Pharmacol. 2000, 59, 1403-1406.

106. Szolcsanyi, J.; Oroszi, G.; Nemeth, J.; Szilvassy, Z.; Tosaki, A. Endothelin release by capsaicin in isolated working rat heart. Eur. J. Pharmacol. 1999, 376, 247-250.

107. Dutta, A.; Deshpande, S.B. Mechanisms underlying the hypertensive response induced by capsaicin. Int. J. Cardiol. 2010, 145, 358-359.

108. Holzer, P.; Lippe, I.T. Stimulation of afferent nerve endings by intragastric capsaicin protects against ethanol-induced damage of gastric mucosa. Neuroscience 1988, 27, 981-987.

109. Szolcsanyi, J. Effect of capsaicin, resiniferatoxin and piperine on ethanol-induced gastric ulcer of the rat. Acta Physiol. Hung. 1990, 75 (Suppl.), 267-268.

110. Mozsik, G.; Szolcsanyi, J.; Racz, I. Gastroprotection induced by capsaicin in healthy human subjects. World J. Gastroenterol. 2005, 11, 5180-5184.

111. Kawada, T.; Hagihara, K.; Iwai, K. Effects of capsaicin on lipid metabolism in rats fed a high fat diet. J. Nutr. 1986, 116, 1272-1278.

112. Watanabe, T.; Kawada, T.; Yamamoto, M.; Iwai, K. Capsaicin, a pungent principle of hot red pepper, evokes catecholamine secretion from the adrenal medulla of anesthetized rats. Biochem. Biophys. Res. Commun. 1987, 142, 259-264.

113. Backonja, M.M.; Malan, T.P.; Vanhove, G.F.; Tobias, J.K. NGX-4010, a high-concentration capsaicin patch, for the treatment of postherpetic neuralgia: a randomized, double-blind, controlled study with an open-label extension. Pain Med. 2010, 11, 600-608.

114. Tesfaye, S. Advances in the management of diabetic peripheral neuropathy. Curr. Opin. Support. Palliat. Care. 2009, 3, 136-143.

115. Sawynok, J. Topical analgesics in neuropathic pain. Curr. Pharm. Des. 2005, 11, 2995-3004.

116. Derry, S.; Lloyd, R.; Moore, R.A.; McQuay, H.J. Topical capsaicin for chronic neuropathic pain in adults. Cochrane Database Syst. Rev. 2009, CD007393.

(C) 2011 by the authors; licensee MDPI, Basel, Switzerland. This article is an open access article distributed under the terms and conditions of the Creative Commons Attribution license (http://creativecommons.org/licenses/by/3.0/). 